\title{
Linking up learners of Chinese with native speakers through WeChat in an Australian tertiary CFL curriculum
}

Wenying Jiang ${ }^{*}$ (D) and Wei Li

\author{
*Correspondence: w.jiang2@uq. \\ edu.au \\ School of Languages and Cultures, \\ the University of Queensland, QLD, \\ St. Lucia 4072, Australia
}

\begin{abstract}
Under the task-based language teaching (TBLT) framework, this study investigated learners' perceptions of a WeChat task being integrated into a Chinese as a foreign language (CFL) curriculum. The WeChat task linked up learners of Chinese from an Australian university with native speakers of Chinese as international students on the same campus. The learner-native speaker pair were required to complete weekly assigned communication sub-tasks through WeChat for ten weeks in semester 2 of 2017. Both a questionnaire survey and an interview were conducted for data collection. Findings show that participants were very positive with the WeChat task. They expressed their enjoyment and satisfaction in completing the question-asking and answering interactions with native speakers through WeChat. As an important component of the tertiary CFL curriculum, the WeChat task was a great learning task to supplement classroom learning. It is also an ideal learning task that makes use of the native speaker resources and mobile assisted language learning technology to achieve the best learning outcome in teaching Chinese as a foreign language.
\end{abstract}

Keywords: Chinese as a foreign language (CFL), WeChat, Task, Mobile assisted language learning

\section{Introduction}

The past three decades have witnessed a surge of interest in task-based language teaching (TBLT), which is evidenced by numerous published monographs, edited volumes and articles in major second language acquisition and language teaching journals (Ellis 2003, 2009; Skehan 1996; Van den Branden 2016; Willis and Willis 2007). Recently a task-supported approach has gained popularity due to the fact that this approach does not take 'task' as the central organizing unit of classroom activity from beginning to end. Instead, tasks are integrated within classroom activity to complement a conventional language-focused curriculum. In particular, a language learning task that makes use of native speaker resources and smartphone technology is intrinsically motivating for learners nowadays. When one learns a foreign language, he or she normally has an urge to use it with a native speaker. Smartphone technology makes it possible to link up learners with native speakers virtually if not physically.

It is anecdotal knowledge that an adult foreign language (FL) learner makes the quickest progress if he or she falls in love with a native speaker of the target language

(c) The Author(s). 2018 Open Access This article is distributed under the terms of the Creative Commons Attribution 4.0 International License (http://creativecommons.org/licenses/by/4.0/), which permits unrestricted use, distribution, and reproduction in any medium, provided you give appropriate credit to the original author(s) and the source, provide a link to the Creative Commons license, and indicate if changes were made. 
(TL). This phenomenon demonstrates the important role of interacting with native speakers in learning an FL. However, most FL learners are taught in a classroom setting, where exposure to the TL is not only limited, but also very often deprived of its culture. In other words, the majority of FL learners do not have the opportunity to visit the country or use the language with native speakers. Although no one would deny the benefits for an FL learner to talk to native speakers, how to approach a native speaker and whether the learner has confidence to do so are obviously the hurdles. When learners do not have a high level of proficiency and confidence in their TL, few of them will seek opportunities to interact with native speakers independent of the language curriculum (Ogawa 1998; Yorozu 2001). Therefore, creative teachers have been seeking various channels to link up their FL students with native speakers outside classroom. With the development of technology and trends of globalization, it is highly possible to extend FL learning from limited classroom talk to real-life interactions with native speakers.

In fact, it is not only possible but also highly popular in employing mobile assisted language learning tasks to enrich an FL curriculum. This is because our FL learners are enthusiastic about using smartphone social communication apps. Unconstrained by time and space, mobile devices offer learners new ways of language learning. Under TBLT framework and using the task-supported approach, this study designs a learning task linking up learners of Chinese with native speakers of Chinese through WeChat, a free Chinese social media mobile app software, and also investigates how well the task is received by these learners of Chinese in an Australian university. Before presenting the theoretical framework of this study, research on WeChat use for language teaching is critically reviewed below.

\section{Review of studies using WeChat for language teaching}

What is WeChat?

WeChat, known as weixin (微信) in Chinese, is the most popular mobile app developed by Tencent in China in 2011. It was originally invented for sending voice messages. Now it has become a multi-functional social networking platform. It allows users to directly text and call each other or conduct a group chat. It also allows users to build an online community with friends by posting texts, photos, and video clips on their own 'moments' (like the news feed on Facebook). Due to its multi-functions, WeChat has skyrocketed in popularity among its users. While WeChat is primarily used in mainland China, it is also used by100 million people (mainly with Chinese ethnicity) in countries outside of China. By 2017, there had been over 938 million active users of WeChat in mainland China and overseas (Wikipedia 2017).

Wang et al. (2016) have explained the rationale in employing WeChat in a foreign language curriculum:

WeChat was chosen as a medium for the language exchange for a number of pedagogical reasons. It is a free application, which literally means micro message. As an instant messenger, it offers potential for supporting asynchronous, semisynchronous and synchronous collaboration and interaction, as it allows for one-toone, one-to-many and many-to-many text and voice messages to be exchanged in real time. Its latest version also supports live one-on-one video conferencing. 
WeChat also facilitates ubiquitous learning as it can be installed on smartphones, laptops, desktop computers and other handheld devices. It can be used as a platform for sharing large amounts of information via text, audio and video files with both individuals and groups, leaving a short message, or starting a conversation both through text and audio chat. The chat can be facilitated by photos, stickers, videos and links, all on the same interface (p. 22).

With so many advantages, there is no reason for language teachers not to consider using WeChat in their teaching. The concern is how to use it effectively and appropriately and not let WeChat distract learners from classroom learning. Previous studies have investigated the use of WeChat as a platform for teachers to give instructions, for providing oral feedback, for managing studying abroad programs and for connecting learners with native speakers.

\section{WeChat used as a platform for teachers to give instructions to students}

Wang's (2015) study discussed the use of WeChat in teaching Chinese as a second language (CSL) in China. In her study, WeChat was mainly used by teachers to give instructions to their students. Twenty students participated in a quasi-experiment with a pre-test/post-test design. The results showed that learners were satisfied with the WeChat-assisted Chinese learning environment and that learners' Chinese proficiency improved rapidly. At the same time, the WeChat platform provided students with a convenient and flexible venue for them to ask questions to satisfy their individual needs. However, the weakness of Wang's (2015) study lies in the fact that no control group was employed. It is hard to tell how much contribution WeChat made in the learners' Chinese proficiency improvement.

\section{WeChat used for teachers to provide oral feedback to learners}

$\mathrm{Xu}$ et al. (2017) used WeChat to provide oral feedback to learners of English as a foreign language (EFL) class. They particularly investigated learners' perceptions of mobile phone assisted oral feedback using the WeChat app. In their study, the instructors gave students feedback via WeChat on their oral production in terms of pronunciation, grammar, content and vocabulary in the forms of both corrective feedback and positive feedback. Data were collected through questionnaires, learners' reflection forms and interviews. Their findings show that learners held positive attitudes towards receiving WeChat feedback and also confirmed the facilitative role of WeChat feedback in enhancing their speaking abilities.

With a similar research design, $\mathrm{Xu}$ and Peng (2017) investigated the use of WeChat in providing oral feedback in teaching Chinese as a second language (CSL) in China. They particularly examined the characteristics of the WeChat-assisted feedback on learners' oral production and learners' perceptions of oral feedback given through WeChat. The results show that the WeChat corrective feedback was centred on overt correction, mainly targeting the linguistic aspects of grammar and vocabulary, whereas positive feedback was concentrated on affirmation, with content and vocabulary as the most frequently focused linguistic targets. In line with the findings of $\mathrm{Xu}$ et al. (2017), $\mathrm{Xu}$ and Peng (2016) also confirmed participants' positive attitudes towards WeChat feedback and its facilitative role in enhancing their speaking ability. 
Although both studies indicate that teachers can use WeChat to provide oral feedback on their students' oral production either in EFL or CSL teaching, students' target language proficiency must be at advanced level for them to understand such oral feedback. Otherwise, the oral feedback given in the target language would mean nothing if students cannot comprehend it. In other words, WeChat needs to be used differently when learners' proficiency level is lower.

\section{WeChat used for managing studying abroad programs}

Jin's (2017) study examined WeChat use by two American university learners of Chinese in a studying abroad program in Shanghai. She adopted the term 'affordance' as a theoretical and analytic construct in investigating the functions of WeChat in students' learning of Chinese during their seven weeks' immersion program in China. 'Affordance' refers to the perceived opportunities for action that an environment provides to a language learner. From this perspective, second language (L2) development only occurs when the resources available in the environment resonate with learners' skills and abilities. Following van Lier's (2004) explanation, 'affordance', in contrast to 'input', emphasizes the learner's agency in perception and action. Affordance is dynamic and emerges out of the interactions between the learner and the environment.

Jin's study identified the following four affordances of WeChat use during the period of the two learners stayed in China: Affordance 1- a casual space with easy access to native speakers of Chinese; Affordance 2 - authentic meaning-focused communication with native speakers of Chinese; Affordance 3 - linguistic resources and multiliteracies; and Affordance $4-$ space for new identity creation.

In sum, Jin found that the WeChat platform was very helpful for the two learners to improve their target language proficiency. In her own words, "As a ubiquitous communication tool in Chinese-speaking communities, WeChat can be integrated in Chinese language education in both domestic and study abroad contexts to connect language learners at various levels of proficiency with native speakers of Chinese" (2017, p. 20). However, her study focused on WeChat use in a seven-week intensive in-country study context in Shanghai. More research needs to be conducted to investigate the potential of integrating WeChat communication in a foreign language context, where a real-life target language practice opportunity is hardly available.

\section{WeChat used for linking up learners with native speakers}

Wang et al. (2016) investigated the use of WeChat as a platform linking up learners of Chinese in Australia with native speakers of Chinese as learners of English in Taiwan. They firstly defined the interactions on WeChat as semi-synchronous, which is between synchronous and asynchronous, in terms of the degree of interactivity and instantaneity of the communication supported by online tools. In their study, the two cohorts of learners were native speakers of each other's target language. In total, five open-ended mega tasks were assigned to students. Each mega task consisted of three sub-tasks, namely a recorded speech, a semi-synchronous dialogue and a form-focused feedback from language partners. The researchers evaluated the affordances of WeChat in a teaching Chinese as a foreign language (CFL) curriculum under a community of inquiry (CoI) framework. The findings from their study confirm the establishment of teaching, 
social and cognitive presences, but with features specific to WeChat-supported semi-synchronous language exchange. They also suggest that "WeChat could be a useful tool in supporting language learning and its potential needs to be further explored" (p. 34). However, the task designed in their study required learners to have advanced proficiency levels of the target language. Then what types of WeChat tasks are appropriate for intermediate or lower intermediate learners of Chinese?

Up to now no empirical research has been conducted to examine the use of WeChat in linking up lower proficiency learners of Chinese with native speakers of Chinese in a Chinese as a foreign language (CFL) curriculum for learners to develop their oral skills and cultural awareness in a relaxing and informal environment outside classroom. It is this group of learners who urgently need to practise their Chinese with native speakers of Chinese but have no confidence in reaching out on their own. To help this cohort of learners and also to bridge the research gap, the present study endeavours to design a WeChat task for lower intermediate CFL learners and also to examine how well the newly designed WeChat task is received by the learners. Measures to improve the management of the task are also investigated so that learners can have a better learning experience.

In sum, the objectives of the study are two folds: (1) to investigate how well the WeChat task is perceived by this group of CFL learners; (2) to elicit suggestions or measures to further improve the management of the WeChat task from the learners' perspective. The rationale in investigating learners' feelings and perceptions lies in the fact that for any learning to occur, it must be through the effort of the learner, or the agency of learning. It is important, therefore, to investigate how learners feel about a learning task, particularly a WeChat task when it is used for the first time. Given that a newly designed WeChat task is at an explorative stage, it is crucial to collect information on how students perceive it and how to enhance agency of learning by having learners' voice heard. Given that the design of the WeChat task in this study is guided by task-based language teaching (TBLT) framework, it makes more sense to elaborate the theoretical framework first before moving on to the study.

\section{Theoretical framework}

The central concept in task-based language teaching (TBLT) framework is the definition of 'task'. Various definitions of 'task' have been proposed by different scholars over the past three decades. As den Branden (2016) has summarized, all the definitions "share a common core: a task is a goal-oriented activity that people undertake and that involves the meaningful use of language" (p. 240). That is to say, when learners are completing a task, they mainly focus on how to achieve a communicative goal in the target language rather than learning grammar rules. Using the target language in a meaningful way is motivating for the learners. That is why the TBLT approach has become popular among second language teaching professionals.

The most frequently cited framework of TBLT is the three-stage model which consists of pre-task activities, the actual task performance and post-task activities (Ellis 2003, 2009; Skehan 1996; Van den Branden 2016; Willis and Willis, 2007). In the pre-task stage, teachers provide instructions to prepare students to perform the task in the ways it is required. Scaffolding measures such as training students to use a particular computer program or helping students to form a pair or a group or answering students' questions in regard to the task assigned or giving an example showing how the task is 
supposed to be conducted are provided during this stage. During the actual task performance stage, most teachers set a time limit, so they can check students' performance after the deadline. On the other hand, teachers also need to give students some freedom to complete the task in their own time. For example, a teacher assigns a semester task that involves weekly sub-tasks. To complete the sub-task each week, students need to commit themselves for two hours. During the whole week, students can choose to do it anytime they like. Teachers can elect to provide feedback each week or at the end of the semester. The post-task stage mainly involves three pedagogic goals: (1) to report the task outcomes, (2) to encourage students to pay attention to form, in particular errors made by the L2 learners when they performed the task, and (3) to repeat performing the task.

One weak point in this TBLT framework is the insufficient attention to the agency of learning in the post-task stage (den Branden 2016). The notion of agency refers to the hypothesis that tasks do not cause learning but are useful vehicles for launching the agency of second language learners in the purposeful practice of communicative language use and verbal interaction. The learner is the agent of his or her own learning. Without seeking feedback from the learner or the agent, namely without examining how well a task is received by the learner, the cycle of the three-stage model is incomplete. Agency of learning is of central concern to the field of education. Schwartz and Okita (2006, p.3) used Table 1 to demonstrate learning tasks that embody high agency and low agency respectively:

In order to enhance agency of learning, teachers should consider all the descriptive properties on the left-hand side when they organize learning tasks for their students. This is because increasing student agency leads to superior and more effective learning (Schwartz and Okita 2006). Aiming to enhance student agency, the authors of the current study have revised the existing TBLT framework by adding a component in the post-task stage seeking learners' feedback on task management to further emphasize agency of learning, which is demonstrated in the figure below:

For a task to be successfully managed and well received, it may require several cycles of the three stages demonstrated above, particularly requiring investigation on how learners feel about the task and what improvements can be made for future use of similar tasks.

\section{Method}

\section{The design and management of the WeChat task}

Following Tian and Wang's (2010) recommendation that social software can take "part of the learning outside the classroom and into the real world" (p. 194), the authors of

Table 1 Contrasts of high agency versus low agency learning tasks

\begin{tabular}{ll}
\hline High Agency & Low Agency \\
\hline Student Centered & Teacher Centered \\
Student Voice & Authoritarian \\
Constructivism & Transmission \\
Active & Passive \\
Doing & Watching \\
Elective & Compulsory \\
Intent Participation & Assembly Line \\
In Control & Programmed \\
\hline
\end{tabular}


the current study included a newly designed WeChat task in their Chinese as a foreign language (CFL) curriculum. In the second semester of 2017, a WeChat task linking up learners with native speakers was integrated into the Second Year Spoken Chinese (CHIN3010) language course offered by the Chinese program at a well-known university in Australia. Based on the revised TBLT framework demonstrated in Fig. 1 above, namely the three-stage model, the pre-task stage and the task performance stage are presented below. Data collection of this study focused on the post-task stage, which means that after completing the task, learners' feelings and perceptions on the task and task management were elicited in order to justify and improve the running of this learning task.

In Teaching week 1 of the second semester of 2017, CHIN3010 students were required to download a WeChat App and install it on their smartphones. Steps of doing so were spelled out and provided to students in class. At the same time, the instructors recruited a group of Chinese native speakers on campus who were international students wishing to have opportunities to practice their English with Australian native speakers of English. After all the learners of Chinese had installed WeChat to their smartphones, each of them was paired up through WeChat with one of the Chinese native speakers recruited. Each pair was regarded as language partners to each other. The instructors/researchers joined each pair in WeChat groups in order to monitor their interactions.

The university has two 13-week-teaching semesters each year. During second semester Teaching weeks 2 to 11, 10 weeks in total, a weekly communication topic (see Table 2) was assigned to the students. The native speaker and the learner were required to ask each other five questions in Chinese based on the topic assigned weekly. The questions asked must be understood and relevant answers must be successfully elicited. In other words, a student of Chinese must ask his or her language partner (a native speaker of
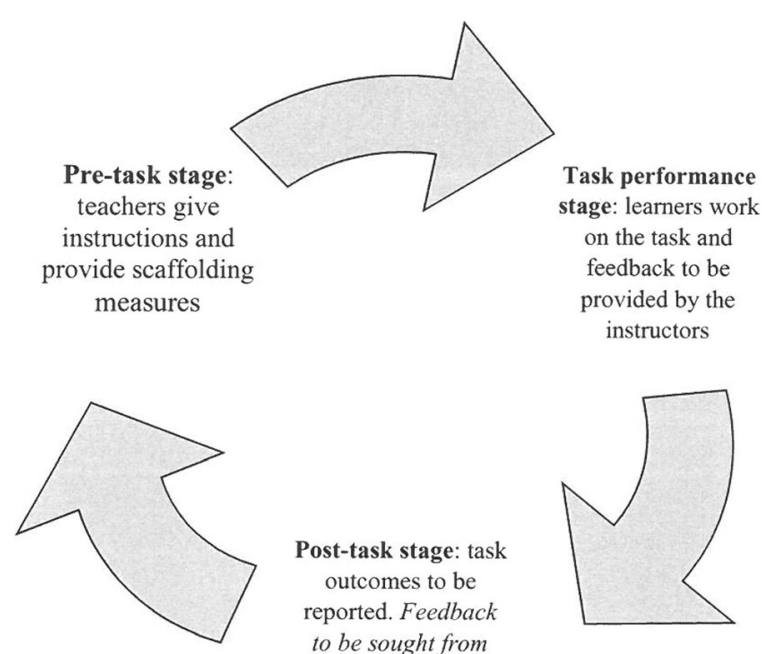

Post-task stage: task outcomes to be reported. Feedback to be sought from learners to improve task management

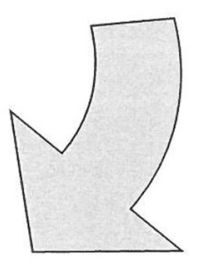

Fig. 1 Revised framework of task-based language teaching. The figure shows the dynamic cycle of the three stages, namely pre-task stage, task performance stage and post-task stage, in task-based language teaching. The management of a particular task will be enhanced through seeking feedback from the learners at the past-task stage. For a task to be successfully managed and well received, it may require several cycles of the three stages 
Table 2 The weekly topics during the 10 weeks of task performance

\begin{tabular}{llll}
\hline Teaching Week & Topic assigned & Teaching Week & Topic assigned \\
\hline 2 & Self-introduction & 7 & Name choosing \\
3 & Language learning experience & 8 & Chinese festivals \\
4 & Hobbies & 9 & The person you admire most \\
5 & Travel & 10 & Part-time work or interview \\
6 & Your hometown & 11 & Who pays your bills (rent, \\
& & & mobile phone, tuition etc.) \\
\hline
\end{tabular}

Chinese) five questions, get a relevant answer to each question and at the same time she/ he must answer each of the five questions that the native speaker asks. The question-answer interactions should be recorded on WeChat as voice messages in each individual WeChat group, in which the instructors were silent listeners. For the learners, practice before the recording was not only allowed but also encouraged.

The rationale of this design, namely asking each other questions, was to make sure that learners had effective interactions with native speakers. Due to their limited proficiency level, learners would be at loss if native speakers talked freely and consecutively. By asking each other questions, learners were well-targeted in receiving and comprehending the voice messages. Moreover, leaners could play native speakers' voice messages as many times as they wished. They could also prepare and rehearse before asking and answering questions in order to reduce the anxiety. They could also delete their voice messages after posting and re-do it if they were not yet satisfied.

All the communication sub-tasks assigned weekly were related to the lessons covered in the previous week. Hence learners could make use of the vocabulary and sentence structures that they had just learnt. The interactions between learners and native speakers were monitored by the instructors/researchers. On Monday of each week during weeks 2 to11, the instructors reminded the learners of the topic in that week. They also gave examples in regard to what types of questions could be asked. The topics for the 10 weeks are presented in Table 2 .

As part of the course curriculum, the WeChat task was allocated with a $15 \%$ assessment weighting. It was stated in the course profile that the marking criteria only included clarity of the message recorded on WeChat and timely completion of the interactions. In order to encourage students to talk as much as they can, errors in pronunciation, tones, vocabulary and grammar are not penalized. The rationale lies in the point that speaking skills are learnt or acquired through speaking. The deadline was set at midnight Sunday in teaching weeks $2-11$ with Monday being the first day of each week. For example, week 2 recording was due at midnight on Sunday 6 August 2017. The task-performance stage lasted from 31 July to 15 October 2017, 10 weeks in total. Each week learners performed a question-asking and question-answering communication sub-task with their Chinese native speaker language partners based on the topic assigned.

\section{Participants}

Fifteen students who enrolled in the Second Year Spoken Chinese (CHIN3010) course in 2017 at the university participated in the study. Eight were female while seven were male. Their average age was 23. The learner cohort for this course was diverse, 
reflecting the contemporary Australian tertiary community demographic, i.e. a broad range of ethnicity, English language competency, computer literacy, age and gender was represented. They had studied Chinese for 1.5 years by the time they were assigned the WeChat task. At the end of the semester the majority reached new HSK (stands for Hanyu Shuiping Kaoshi, the Chinese proficiency test for L2 Chinese learners) level 3 proficiency level.

\section{Instruments}

Two instruments were adopted to investigate how well the WeChat task was received by the students. One is a questionnaire survey (see Additional file 1: Appendix A) with a 5-point scale on statements of how learners felt about the WeChat task. The statements in the questionnaire used in the study were based on Kim (2014)'s study and revised in accordance with the purpose of the current study.

The other one was an interview, which consisted of seven questions designed to find out learners' perception of the task and whether they had any suggestions in the management of the WeChat task. The seven questions for the interview (see Additional file 1: Appendix B) were revised from Lee's (2004) study.

The questionnaire and interview both focused on learners' perceptions of the WeChat task, which was integrated to CHIN3010 as part of the assessment of the second-year spoken Chinese course. The content or the language use of the learner-native speaker interactions in Chinese on WeChat are not the focus of this study, although the interactions have provided rich information in regard to issues of WeChat affordances and feedback provision, etc. Instead, this study focuses on how students perceive the WeChat task in general.

\section{Data collection procedure}

In Teaching week 7 after students had completed six topics, a questionnaire that aimed to urge students to reflect on the WeChat task was distributed in class during the last $15 \mathrm{~min}$. It turned out that 12 students completed the questionnaire. Three students were absent that day.

At the end of the semester, participants were interviewed individually with seven semi-structured questions. The seven questions were designed to investigate how well the WeChat task was received by the students and how to improve the management of the task if it were to be employed again in the following year. The interview with each participant lasted about $10 \mathrm{~min}$. All 15 students participated in the interview on Friday 17 November 2017. The interview was audio recorded, transcribed by the first author, and checked by the second author.

The questionnaire survey and the semester interview were both conducted in English. Due to their limited proficiency level in Chinese, English was used to ensure that students fully understand what was asked and accurate responses be provided.

\section{Results}

\section{Questionnaire survey in week 7}

The first part of the questionnaire survey included seven statements requiring participants to choose among the 5-point scale degrees: 5 indicates 'Strongly agree', 4 'Agree', 
3 'Neutral', 2 'Disagree' and 1 'Strongly disagree'. The mean scores and standard deviations received are summarized in Table 3.

As demonstrated in Table 3, the results show that the mean scores of the seven statements are close to or above 4 respectively, which means that participants generally agree to all the statements. The highest score achieved is 4.58 to the statement "The WeChat task provides me opportunities to use Chinese". The second highest score is 4.42 to the statement "The WeChat task helps me to connect learning Chinese with real-world situations". These are the principal goals for the WeChat task to achieve. At the same time, participants agree that the instructors have provided scaffolding measures for them to use WeChat (scored 4.33) and the WeChat task enhanced their confidence in using the target language Chinese (scored 4.08). The lowest average score achieved is 3.92 for both statements "The way the WeChat task is organized is great for me to use Chinese" and "The WeChat task has enhanced my interest in learning Chinese". This might indicate that the WeChat task could be organized better and it could further enhance learners' interest in learning the target language.

The average time that participants spent on the weekly WeChat sub-tasks ranges from 20 min to four hours. The group's average time spent weekly was $88 \mathrm{~min}$, close to $1.5 \mathrm{~h}$. This shows that students' investment of time and effort on this task vary greatly at an individual level. Consequently, students might have benefited at varying degrees from this task. It is likely that the more time and effort they committed to the task, the more they benefited.

In regard to ways of improving the management of the WeChat task, eight out of the 12 participants provided at least one suggestion. They are:

(1) "I like more basic every day topics";

(2) "allow students to conduct the task with their existing Chinese friends if they have any";

(3) "let the native speaker language partner know the weekly topics, so they stick to the topic with relevant questions";

(4) "I wish we were given more clear instructions about how long we should hold the conversation";

(5) "rather than just giving us the topics/instructions for each week, native speakers should also be trained or informed before the commencement of this assessment";

(6) "some topics are too broad; more details would be helpful as it sometimes goes off topic during our conversation";

(7) "train native speakers to speak slowly"; and

Table 3 Mean scores and standard deviations of the seven statements

\begin{tabular}{lll}
\hline Items & Mean & SD \\
\hline I learn a lot through the WeChat task with native speakers & 4.00 & 0.739 \\
The WeChat task helps me to connect learning Chinese with real-world situations & 4.42 & 0.669 \\
The WeChat task provides me opportunities to use Chinese & 4.58 & 0.669 \\
The instructors have provided scaffolding measures for me to use WeChat & 4.33 & 0.888 \\
The way the WeChat task is organized is great for me to use Chinese & 3.92 & 0.669 \\
The WeChat task has enhanced my confidence in using Chinese & 4.08 & 0.669 \\
The WeChat task has enhanced my interest in learning Chinese & 3.92 & 0.793 \\
\hline
\end{tabular}


(8) "I would like my language partner to be more active".

Among all the suggestions above, no. (3) and no. (7) were somewhat remedied after week 7. As the course instructors, we reminded the native speaker group that it would be more helpful if they could stay to the weekly topics (3) and if they could speak slowly to accommodate our learners (7). Based on the experience, it is believed that for the future use of such a WeChat task for CFL learners at this level of proficiency, the native speaker group needs a training session. They need to be trained to speak more slowly, and to be more active and more considerate to the learners. Minimum time commitment to the weekly task needs to be spelled out for both learner and native speaker groups. Before assigning a learner a native speaker language partner, the instructors should consider giving them options to work with their own Chinese friends if they have any, which seems more respectful to students and also more motivating.

\section{End of semester interview}

During the interviews, the participants confirmed the positive role of WeChat in facilitating their learning of spoken Chinese. Most of them expressed their excitement in talking in Chinese with native speakers through WeChat because WeChat platform made it convenient to do so. In addition, most of them rarely had any opportunities to use the target language in real life situations otherwise. They confirmed that they had a great experience talking to their native speaker language partners via WeChat. They considered the WeChat task as an innovative and interesting way to supplement classroom learning in general.

When prompted for the most valuable and interesting part of the WeChat task, participants cited the following aspects. P stands for participants.

"My language partner talked about cultural aspects that I cannot really learn in classroom" (P1)

"I feel that I used the target language in a practical sense" (P2, P3e \& P7)

"My language partner and I get to know each other and we have become friends" (P4)

"I got a chance to talk to a native speaker in Chinese. It was exciting to use Chinese in a real life situation." (P5 \& P6)

"I find it valuable in recording my own voice, listen to it, and delete it when I am not happy about it. I can always record it again until I am satisfied" (P8)

"It is a good experience in using Chinese in a practical sense" (P9)

"I feel it is a real communication. It is different from role-plays in classroom"

(P10 \& P11)

"The experience of talking to a native speaker in Chinese is very exciting" (P 12)

"Using the language outside classroom is interesting" (P13, P14 \&15) 
In sum, the participants appreciate the opportunity of using the target language outside classroom with native speakers.

When asked about the most difficult or least important part of the WeChat task, 6 out of the 15 participants (40\%) could not think of anything negative. They confirmed that they greatly enjoyed the task. Three students $(20 \%)$ mentioned that the difficult part was that their native speaker partners spoke too fast that they had to play the voice messages several times to be able to comprehend them. In addition, they said sometimes their native speaker language partners spoke sentence after sentence without a pause, which was quite challenging for them to follow smoothly. Two other students (13\%) commented that it was a bit hard to start a conversation at the very beginning. One participant $(7 \%)$ mentioned that her native speaker language partner had a different accent from that of the teachers, which brought some difficulty in understanding the voice messages. One (7\%) said that she found it hard to ask good questions. Another student $(7 \%)$ expressed his wish that his native speaker partner stay with the topics assigned because he found that his native speaker language partner sometimes strayed from the topics. The last point mentioned (7\%) was that sometimes native speakers used vocabulary that the learner did not know. The learner had to check a dictionary or ask friends for help.

Despite the reported minor challenges, all the participants unanimously confirmed that they enjoyed the interactions with native speaker language partners. In fact, five out of the 15 students, namely one third said "Yes, I really enjoyed it". When asked why, three reasons were cited. The first one is that they appreciated the opportunity to talk to a native speaker of the target language. The second one is that they liked using the target language in a real life situation, namely outside classroom. The third is that getting to know a person, a university mate of similar age, was an enjoyable experience itself.

In regard to the weekly assigned topics, participants all agreed that they were good because each week's topic was related to the lesson just covered in the previous week. The WeChat task provided an opportunity for them to use the words and sentence structures learnt in class. Exchanging questions and answers orally with native speakers served a very good way to review the lessons. In one of the participants' own words, "I really like the dynamics of practising the topic with my language partner via WeChat after we learnt it in class. Just before I forgot the words, I would need to use them again". In sum, students were very positive in regard to the WeChat topics.

Question number 6 in the interview asked whether they think the WeChat task has helped them improve their Chinese. Among the 15 participants, 10 (67\%) said "Yes, it has", whereas 5 (33\%) used the word "definitely" or "It definitely has" in responding to the question. Three participants mentioned that their pronunciation and tones had been improved specifically. Another three said they had become more confident in speaking Chinese. Some said that they had learnt more new words outside classroom by chatting with native speakers and others expressed the feeling that as a language learner they liked to be pushed out of their comfort zone or being "forced" to speak Chinese in order to complete the compulsory task. Otherwise, one could not improve their target language skills as expected.

The last interview question was "do you recommend it (namely the WeChat task) to other learners of Chinese?" Among the 15 participants, 13 (87\%) replied firmly that 
they would recommend it. Three of the 13 even said that they would "strongly" or "definitely" recommend it to other learners of Chinese. One student provided a compromised response in saying that "Yes, I recommend it. But I won't recommend it to beginners though". Another student was hesitating in regard to whether he should recommend it because his native speaker language partner was very quiet and he wished he had a different language partner. When prompted why they recommend the task, participants said "putting what you have learnt into practice is really good", "it is important to use the target language outside classroom" and "the task is a good way to supplement our classroom learning". In sum, the interview data strongly support the findings from the questionnaire. Both the questionnaire and the interview data indicate that the WeChat task was a well-received assessment task and the students had a good experience in completing the task.

\section{Discussion and conclusion}

Under the revised task-based language teaching (TBLT) framework, this study examined how well the WeChat task was received when it was used in linking up low proficiency learners of Chinese with native speakers outside classroom. Positive attitudes were found among students, who had expressed their enjoyment and satisfaction in completing the question-asking and question-answering interactions with native speakers through WeChat. In addition, students also gained more confidence in speaking Chinese. This practice compensated for some of the limitations of classroom teaching, where students did not have opportunities to use the target language in real-life situations.

The findings prove that keeping agency of learning high in designing a task is very important. When referring back to the descriptors of high versus low agency in Table 1 quoted earlier, the WeChat task designed in this study has embodied all the descriptive properties listed on the left-hand side, which means that the WeChat task is a high agency learning task. Firstly, the WeChat task is student-centered in that students were supposed to complete the task in their own time. Secondly, while students were completing the weekly communication sub-tasks, the teachers were physically hearing student voice in the target language during the 10 weeks' actual task performance stage. Through the questionnaire survey and the interview, the researchers heard the students' voice, which informed us about how they feel about the task. The third descriptor is constructivism. The WeChat task echoes the arguments proposed by the constructivists in applied linguistics in that the primary function of language is communication rather than representation. Second language learning occurs most effectively during meaningful interactions. The WeChat task enables learners of Chinese to interact with native speakers, which is a good example supporting constructivism. Descriptors Nos. 4 and 5 are active and doing. To complete the WeChat task, students have to be active and doing things in the target language. The task also embodies the notion of elective as students can choose to do it at any time they like during the whole week. They can ask questions that they prefer to ask. In regard to intent participation, when students ask questions of their own choices, they express their intent. Therefore, the WeChat task does promote intent participation. The last descriptor is control. The WeChat task gives students control in many aspects such as when and where they do the task, what specific questions they want to ask, how many times they want to practice their 
questions before posting their voice messages and whether they want to delete any voice messages that they have posted. In sum, given that the task design has considered all the high agency properties, it is not surprising that the WeChat task turned out to be a well-received task.

One criterion for judging whether a learning task embodies low or high agency is whether learners like it and how much they like it. When learners like a learning task very much, it is likely that their agency is high and the learning is more effective. Based on the findings from the questionnaire and interview, the following improvements are to be considered if the WeChat task is used again for learners of low intermediate proficiency level. With the improvements gained through the post-task stage feedback from learners, another cycle of the three-stage model task management can be launched with more confidence.

Firstly, a training session should be provided to the native speaker volunteer group before the learner-native speaker interaction starts on WeChat. Unless a native speaker is a language teacher himself/herself, s/he usually is not considerate enough to accommodate L2 learners' language use. In the interview, three students mentioned that the difficult part of the task was that their native speaker partners spoke too fast. Therefore, the native speaker volunteer group need to be trained to speak slowly, to pause between sentences and to pronounce each word clearly. The main objective of the training is to raise awareness among native speakers for them to be more accommodating. They also need to be aware that learners have limited vocabulary range. In order for learners to follow them smoothly, native speakers need to use words selectively, giving priority to more basic words if they can help it. An encouraging attitude from the native speaker is also highly appreciated because it can make a huge difference to the learner.

Secondly, in the WeChat task instructions, a minimum time requirement needs to be spelled out. Based on the findings from the questionnaire, time spent among the students ranged from 20 min to four hours. It would not be fair if all of them received the same mark in completing the task with such huge differences of commitments. It is recommended that the minimum length of time be spent on the task weekly is one hour, otherwise, the effect of learning can be compromised.

Thirdly, for the language exchange partnership to have a healthy sustainable development, native speaker language partners should also benefit from the WeChat interactions. In the WeChat task instructions in the future, it is recommended to add a friendly reminder that learners of Chinese should also help their native speaker language partner to practice their English. After the native speaker language partner helps the learner of Chinese complete his/her weekly communication task, a similar amount of time should be committed to practising English. Otherwise, the native speaker language partners will gradually lose their enthusiasm in the WeChat interactions.

Finally, in order to keep learners highly motivated in learning Chinese, it is recommended that learners should be given options to work with their own Chinese friends if they prefer, which was suggested and requested by one of the participants. There is no reason for us not to accommodate learners' preferred way of learning.

With the development of smartphone technology, WeChat as a mobile assisted language learning app has received increased attention in foreign language teaching because it is largely free of time and location constraints and highly convenient for 
language learners. This study has provided an instruction model for employing a WeChat task under TBLT framework with task-supported approach in helping FL learners of lower intermediate proficiency level.

\section{Additional file}

Additional file 1: Appendix A. Survey in Week 7 for the CHIN3010 WeChat task. Appendix B. Questions for the semi-structured interview. (DOC $58 \mathrm{~kb}$ )

\section{Abbreviations}

CFL: Chinese as a foreign language; CHIN3010: a course code used in the university where data were collected; Col: Community of inquiry; CSL: Chinese as a second language; EFL: English as a foreign language; FL: Foreign language; L2: Second language; TBLT: Task-based language teaching; TL: Target language

\section{Acknowledgements}

Thanks go to Associate Professor Karen Moni who discussed the design of the study with the authors and offered survey samples for the authors' reference.

\section{Funding}

The current study is part of a larger project entitled "Strengthening the gateways: Building pathways to success through active learning/Active Learning Pathways Project (ALPP)" led by Associate Professor Karen Moni in the Faculty of Humanities and Social Sciences at the University of Queensland (UQ), Australia. The funding sponsored the second author for collecting and analysing the survey data and finding relevant literature because she works as a casual staff member at UQ.

\section{Availability of data and materials}

The data is stored in the first author's computer. Please contact author for data requests.

\section{Authors' contributions}

WJ and WL designed the study together. WL collected and analysed the questionnaire data while WJ collected and analysed the interview data. WJ drafted the first version of the manuscript after discussing with WL. WL read the whole draft of the first version and made some changes in literature review and the interpretation of the findings, which resulted in the second version. Then WJ revised the second version after two weeks, which resulted in the third version. Both authors read and approved the final manuscript.

\section{Authors' informations}

Dr. Wenying Jiang is a lecturer in Chinese in the Chinese program in the School of Languages and Cultures at the University of Queensland. Her research interests include second language acquisition of Chinese, Chinese pedagogy, technology enhanced language learning and intercultural communication.

Dr. Wei Li is currently a Chinese language instructor in the Chinese program in the School of Languages and Cultures at the University of Queensland. Her research interests include pragmatics, interlanguage and intercultural pragmatics, teaching Chinese/English as a second/foreign language, technology enhanced language learning and translation studies.

\section{Competing interests}

The authors declare that they have no competing interests.

\section{Publisher's Note}

Springer Nature remains neutral with regard to jurisdictional claims in published maps and institutional affiliations.

Received: 15 March 2018 Accepted: 31 July 2018

Published online: 16 August 2018

\section{References}

den Branden, V. (2016). Task-based language teaching. In G. Hall (Ed.), Routledge handbook of English language teaching (pp. 238-251). Routledge: London.

Ellis, R. (2003). Task-based language learning and teaching. Oxford: Oxford University Press.

Ellis, R. (2009). Task-based language teaching: Sorting out the misunderstandings. International Journal of Applied Linguistics, 19, 221-246.

Jin, L. (2017). Digital affordances on WeChat: Learning Chinese as a second language. Computer Assisted Language Learning. https://doi.org/10.1080/09588221.2017.1376687.

Kim, H. (2014). Effects of using mobile devices in blended learning for English reading comprehension. MultimediaAssisted Language Learning, 17(2), 64-85.

Lee, L. (2004). Learners' perspectives on networked collaborative interaction with native speakers of Spanish in the US. Language Learning \& Technology, 8(1), 83-100.

Ogawa, K. (1998). Impact of an interaction-oriented Japanese course on learners. Australian review of applied linguistics, $15,123-138$. 
Schwartz, D. L., \& Okita, S. A. (2006). The productive agency in learning by teaching. Stanford: Stanford University Unpublished manuscript.

Skehan, P. (1996). A framework for the implementation of task-based instruction. Applied Linguistics, 17(1), 38-62.

Tian, J., \& Wang, Y. (2010). Taking language learning outside the classroom: Learner's perspectives of eTandem learning via Skype. The Journal of Innovation in Language Learning and Teaching, 4(3), 181-197.

van Lier, L. (2004). The ecology and semiotics of language learning: A sociocultural perspective. Boston, MA: Kluwer Academic.

Wang, Y., Fang, W.-C., Han, J., \& Chen, N.-S. (2016). Exploring the affordances of WeChat for facilitating teaching, social and cognitive presence in semi-synchronous language exchange. Australasian Journal of Educational Technology, 32(4), 18-37.

Wang, Z. (2015). Weixin pingtai zai yuyan xuexi zhong de yingyong yanjiu (微信平台在语言学习中的应用研究), English translation title: Effects of using WeChat in an experimental CSL program. Open Education Research, 21, $113-118$.

Wikipedia (2017). Retrieved at https://en.wikipedia.org/wiki/WeChat on 21 November 2017.

Willis, J., \& Willis, D. (2007). Doing task-based teaching: A practical guide to task-based teaching for ELT training courses and practising teachers. Oxford: Oxford University Press.

Xu, Q., Dong, X. Q., \& Jiang, L. (2017). EFL learners' perceptions of mobile-assisted feedback on oral production. TESOL Quarterly, 51(2), 408-417.

Xu, Q., \& Peng, H. (2017). Investigating mobile-assisted oral feedback in teaching Chinese as a second language. Computer Assisted Language Learning, 30(3-4), 173-182.

Yorozu, M. (2001) Interaction with native speakers of Japanese: What learners say, Japanese Studies, 21(2), 199-213.

\section{Submit your manuscript to a SpringerOpen ${ }^{\odot}$} journal and benefit from:

- Convenient online submission

- Rigorous peer review

- Open access: articles freely available online

- High visibility within the field

- Retaining the copyright to your article

Submit your next manuscript at $\boldsymbol{\nabla}$ springeropen.com 